\title{
Contribution of flow cytometry to acute leukemia classification in Tunisia
}

\author{
S. Feki ${ }^{a}$, H. El Omri ${ }^{\text {b }}$, M.A. Laatiri ${ }^{\text {, }}$ \\ S Ennabli ${ }^{\mathrm{b}}, \mathrm{K}$. Boukef ${ }^{\mathrm{a}}$ and F. Jenhani ${ }^{\mathrm{a}, *}$ \\ ${ }^{a}$ Laboratoire d'Immunologie Cellulaire, Centre \\ National de Transfusion Sanguine, Tunis, Tunisia \\ ${ }^{\mathrm{b}}$ Service d'Hématologie Clinique, Hôpital Farhat \\ Hached, Sousse, Tunisia
}

Received 14 April 2000

Accepted 11 May 2000

The precision of immunological characterization of leukemias was improved by a certain number of technical innovations, particularly hybridoma production and standardization, resulting in monoclonal antibodies and definition of recognised cellular antigens (designated by CD: Cluster of Differentiation).

The aim of this work was to determine the immunophenotyping profile of patients with leukemia, by means of a flow cytometric method: 66 blood samples coming from leukemic persons in the Sahel region were studied by flow cytometry, using about thirty monoclonal antibodies all marked with a fluorochrome, in one or two colour systems to assess their distribution according to type (lymphoid $\mathrm{B}$ or $\mathrm{T} /$ myeloid) and age, and to search for possible co-expressions of markers of different lineages.

The marked preponderance of childhood B-ALL in our series is, at least partly, attributable to the age distribution of the Tunisian population. In agreement with studies from other countries, the majority of AML cases occurred among adults. A high proportion of AML cases in our series co-expressed markers of other lineages. Overall, accurate classification of acute leukemias was possible from a simple peripheral blood sample in 62 of 66 cases (93.9\%).

Keywords: Acute leukemia, markers, flow cytometry, Tunisia

\footnotetext{
${ }^{*}$ Correspondence: Pr Faouzi Jenhani, B.P. 365, El Menzah 1004, Tunisia. Fax: +2161562957 / 21622766 33; E-mail: faouzi. jenhani@fphm.rnu.tn.
}

\section{Introduction}

At present, characterization and classification of leukemias in the laboratory is carried out on the basis of morphology, cytochemistry, cytogenetics and immunophenotype analysis. The immunological approach was improved by some technical innovations, particularly hybridoma production and standardization, giving rise to monoclonal antibodies, and by the definition of "Clusters of Differentiation" (CD). Application of these reagents became easier following improvement in flow cytometric methods, production of new fluorochromes and the development of labelling techniques applied to cell suspensions and directed to either surface or cytoplasmic molecules.

The assignment of acute leukemias to a given lineage is an important step for determining prognosis and therapy. Several classifications have been proposed, particularly that of the French Groupe d'Etude Immunologique des Leucémies (GEIL) that proposes an immunologic classification of acute leukemias, based on a scoring system where individual markers are assigned major, intermediate and minor weights on a scale of $1.5,1$ and 0.5 points, respectively. Assignment to a given lineage requires a score of at least 2 with the appropriate marker set. Thus, four categories can be defined, "nul", "pure", "variant" and "multiphenotypic" depending on score combinations in the three lineages $\mathrm{B}, \mathrm{T}$ and myeloid [5].

\section{Material and methods}

The aim of this work was to determine the immunophenotypic profile of leukemic patients coming from the Sahel region of Tunisia (North Africa). 66 patients were studied aged from 3 days to 71 years (with a mean of 19.6 years). All had a recent diagnosis of leukemia at Farhat Hospital of Sousse.

Immunophenotyping analyses were performed at the Cellular Immunology Laboratory of the National Center of Blood Transfusion of Tunisia. We used a panel 
Table 1

Number of acute leukemias in terms of type, age and coexpression of other lineage markers

\begin{tabular}{|c|c|c|c|c|c|c|c|c|}
\hline \multirow[t]{2}{*}{ AGE } & \multicolumn{2}{|c|}{ B-ALL } & \multicolumn{2}{|c|}{ T-ALL } & \multicolumn{2}{|c|}{ AML } & \multirow[t]{2}{*}{ Biphénotypic AL } & \multirow[t]{2}{*}{ TOTAL } \\
\hline & Pure & coexpr & Pure & coexpr & Pure & coexpr & & \\
\hline $0-10$ years & 15 & 0 & 7 & 0 & 3 & 1 & 0 & 26 \\
\hline $11-20$ years & 4 & 0 & 3 & 0 & 4 & 2 & 0 & 13 \\
\hline$>21$ years & 2 & 0 & 1 & 1 & 8 & 9 & 2 & 23 \\
\hline \multirow[t]{2}{*}{ TOTAL } & 21 & 0 & 11 & 1 & 15 & 12 & 2 & 62 \\
\hline & \multicolumn{2}{|c|}{21} & \multicolumn{2}{|c|}{12} & \multicolumn{2}{|c|}{27} & & \\
\hline
\end{tabular}

Table 2

Distribution of acute leukemia types according to age

\begin{tabular}{lcccc}
\hline AGE & B-ALL & T-ALL & AML & Biphénotypic AL \\
\hline 0-10 years & $71.4 \%$ & $58.3 \%$ & $14.8 \%$ & $0 \%$ \\
11-20 years & $19.1 \%$ & $25 \%$ & $22.2 \%$ & $0 \%$ \\
$>21$ years & $9.5 \%$ & $16.7 \%$ & $63 \%$ & $100 \%$ \\
TOTAL & $100 \%$ & $100 \%$ & $100 \%$ & $100 \%$ \\
\hline
\end{tabular}

of 29 monoclonal antibodies (see below), a direct immunofluorescence reaction on fresh peripheral blood cells with a red cell lysing method (FACSlysing solution of Becton Dickinson) according to Lees et al. [11] and acquisition on a FACSCalibur flow cytometer (Becton Dickinson) with CellQuest software. Data were expressed as percentages of labelled blast cells; positivity thresholds were $30 \%$ for B-lineage markers, $90 \%$ for T-lineage markers, $20 \%$ for myeloid antigens and $7 \%$ for immaturity markers (CD34, CD117, CD1a and CD10).

Monoclonal antibodies used (Immunotech):

CD1a (PE) - CD2 (FITC or PE) - CD3 (FITC or PE) - TCR $\alpha \beta$ (FITC) - CD4 (FITC or PE) - CD5 (FITC) - CD7 (FITC) - CD8 (FITC or PE) - CD10 (FITC) - CD13 (FITC or PE) - CD14 (FITC or PE) - CD15 (FITC) - CD16 (FITC or PE) - CD19 (FITC or PE) CD20 (FITC or PE) - CD22 (FITC) - CD23 (FITC) - CD33 (PE) - CD34 (FITC or PE) - CD36 (FITC) CD38 (FITC) - CD41 (FITC) - CD45 (FITC) - CD56 (PE) - CD65 (FITC) - CD71 (FITC) - CD117 (PE) HLA DR (FITC) - FMC7 (FITC) - Isotypic controles (Mouse antibodies: IgG1 FITC, IgG1 PE, IgG2a FITC, IgM FITC)

\section{Results and discussion}

Among the 66 samples studied, 62 could be assigned to a given lineage (Table 1); that means that we were able to type an acute leukemia in a simple blood sample, in $93.9 \%$ of cases. The 4 cases for whom immunophenotyping was apparently normal, were patients who had received intensive corticosteroid therapy during the last few days preceding their blood collection.

Among the 62 acute leukemias newly diagnosed and sent to our laboratory, we found 21 B-ALL (33.9\%), 12 T-ALL (19.4\%) 27 AML (43.5\%) and 2 biphenotypic acute leukemias $(3.23 \%)$.

Considering the distribution according to age, 39 cases (62.9\%) were young persons (from 0 to $20 \mathrm{yrs),}$ whereas only 23 cases (37.1\%) were adults (aged 21 or over). This could be explained, in part, by the fact that nearly $2 / 3$ of the Tunisian population are under 30 .

Furthermore (Table 2) and in accordance with the literature [10], $71.4 \%$ of B-ALL and $58.3 \%$ of T-ALL were children under 10 , whereas that age bracket contains only $14.8 \%$ of AML; in fact, the majority of AML $(63 \%)$ are adults over 21.

Moreover (Table 3), within the $0-10 \mathrm{yr}$ age bracket, B-ALL are in the majority (57.7\%), when compared with AML (15.4\%); examining successively older age cohorts the B-ALL proportion decreases $(8.7 \%$ for those over 21) whereas AML increases (73.9\% for those over 21); these observations agree well with the literature [8].

Finally (Table1 ), among the 27 AML cases investigated, $12(44.4 \%)$ coexpress markers of other lineages: $22.2 \%$ (6 cases) coexpress the CD56 marker, which is relatively high in comparison to previous reports (5.71\% according to Scott, 1994$) ; 14.8 \%$ (4 cases) coexpress CD19 (one of which coexpress CD56) and one case $(3.7 \%)$ coexpresses the CD2 marker. Coexpression of CD19 is correlated with AML2 with $t(8,21)$ translocation [9] and according to the CALGB study, CD56 coexpression carries a bad prognosis [1,4]. In non-AML cases, we detected just one instance of coexpression. This was a case of CD13 positiveT-ALL ( $8.33 \%$ of T-ALL). 
Table 3

Distribution of the age groups according to the cellular type

\begin{tabular}{lccc}
\hline & $0-10$ years & $11-20$ years & $>21$ years \\
\hline B-ALL & $57.7 \%$ & $30.8 \%$ & $8.7 \%$ \\
T-ALL & $26.9 \%$ & $23.1 \%$ & $8.7 \%$ \\
AML & $15.4 \%$ & $46.1 \%$ & $73.9 \%$ \\
Biphénotypic AL & $0 \%$ & $0 \%$ & $8.7 \%$ \\
TOTAL & $100 \%$ & $100 \%$ & $100 \%$ \\
\hline
\end{tabular}

\section{Conclusion}

Over the past twenty years, blast immunophenotyping have become very important in acute leukemia diagnosis. Classification by cytology alone, or even coupled with cytochemistry, can be problematic. This study shows that immunophenotyping by flow cytometry, which has recently become available in Tunisia, permits the assignment of the leukaemic cells present in a given sample to a precise cellular lineage. More interesting is the fact that we could, in a great number of cases, avoid taking a bone marrow sample which could be traumatic for the patient. However, blast immunophenotyping cannot replace cytology; it simply constitutes a valuable complement in the precise diagnostic of acute leukemia and lymphoma.

\section{References}

[1] M.R. Baer, C.C. Stewart and D. Lawrence et al., Expression of the neural cell adhesion molecule CD56 is associated with short remission duration and survival in acute myeloid leugemia with t (8;21) (q22;q22), Blood 90 (1997), 16431648 .

[2] M.-C. Béné, O. Lees and G. Faure et le GEIL, Immunophénotypage des leucémies: recommandations, Revue Française des Laboratoires 287 (1996), 47-52.

[3] J.M. Bennett, D. Catovsky and M.T. Daniel et al., Proposals for the classification of acute leukemias. French-American-
British (FAB). Cooperative Group, Br J Haematol 33 (1976), $451-458$.

[4] J.C. Byrd, R.B. Weiss and D.C. Arthur et al., Extramedullary leukemia adversely affects hematologic complete remission rate and overall survival in patients with $\mathrm{t}(8 ; 21)$ (q22; 22$)$ : results from Cancer and Leukemia Group B 8461, J Clin Oncol 15 (1997), 466-475.

[5] R. Garand and M.-C. Béné et le GEIL, A new approach of acute lymphoblastic leukemia immunophenotypic classification: 1984-1994 the GEIL experience, Leukemia and Lymphoma 13(1) (1994), 1-5.

[6] I. Hannet, F. Erkeller-Yuksel and P. Lydyard et al., Developmental and maturational changes in human blood lymphocyte subpopulations, Immunology Today 13 (1992), 6.

[7] M. Imbert, marqueurs cellulaires des lignées lymphoïdes et myéloïdes normales, Revue Française des Laboratoires 218 (1991), 37-43.

[8] H. Jouault, apport de la cytométrie en flux à la caractérisation des leucémies aiguës, Revue Française des Laboratoires 218 (1991), 79-84.

[9] K. Kita, K. Nakase and H. Miwa et al., Phenotypical characteristics of acute myelocytic leukemia associated with the $\mathrm{t}$ $(8 ; 21)$ (q22;q22) chromosomal abnormality: frequent expression of immature B-cell antigen CD19 together with stem cell antigen CD34, Blood 80 (1992), 470-477.

[10] O. Lees and M.-C. Béné et les membres du GEIL, Immunophénotypage des leucocytes Clusters de différenciation humains. Application à la caractérisation des hémopathies, Biotem Editions, Paris, 1998.

[11] O. Lees, Immunophénotypage des cellules sanguines: du prélèvement à la lecture au cytomètre: les différentes méthodes, Revue Française des Laboratoires 287 (1996), 3339.

[12] Scott et al., Blood 84 (1994), 244-255. 


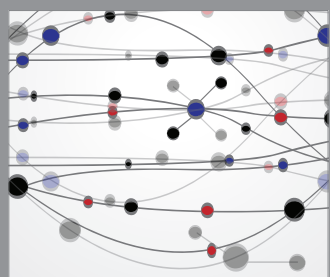

The Scientific World Journal
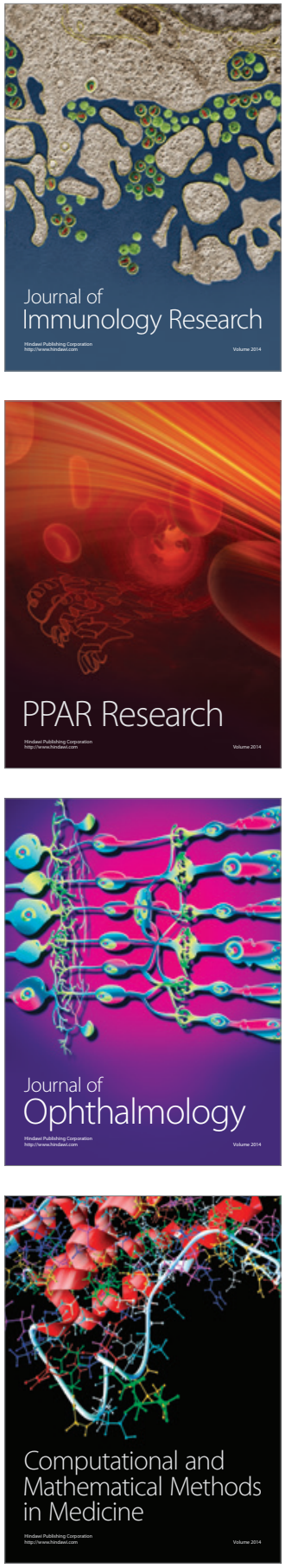

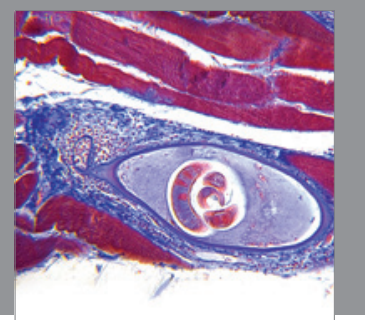

Gastroenterology

Research and Practice
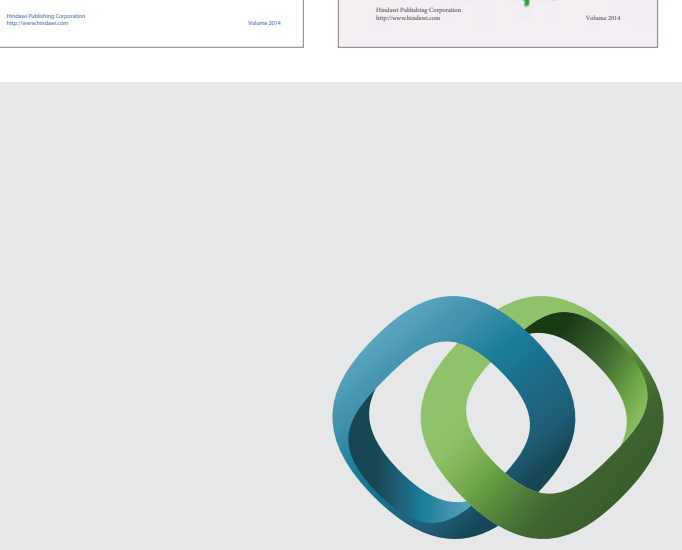

\section{Hindawi}

Submit your manuscripts at

http://www.hindawi.com
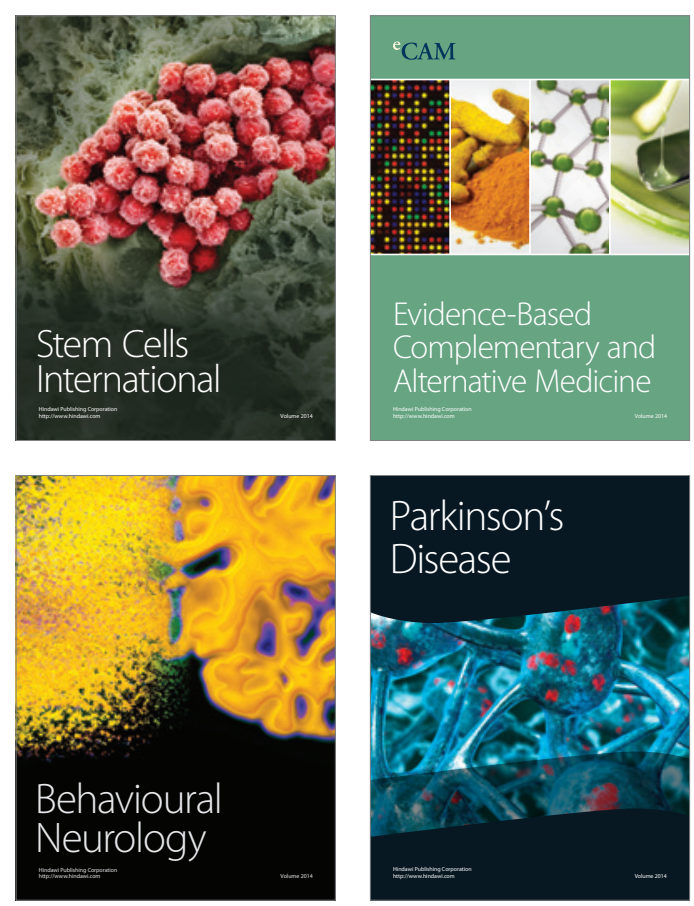

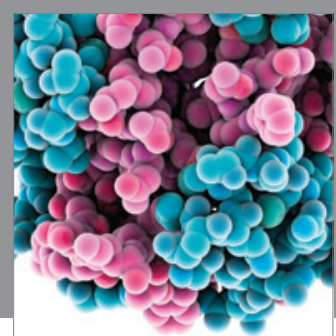

Journal of
Diabetes Research

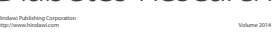

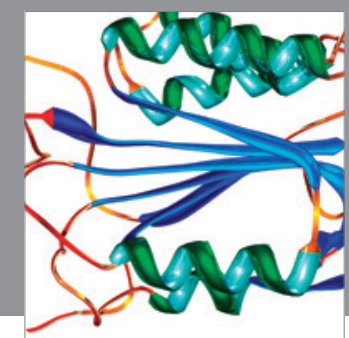

Disease Markers
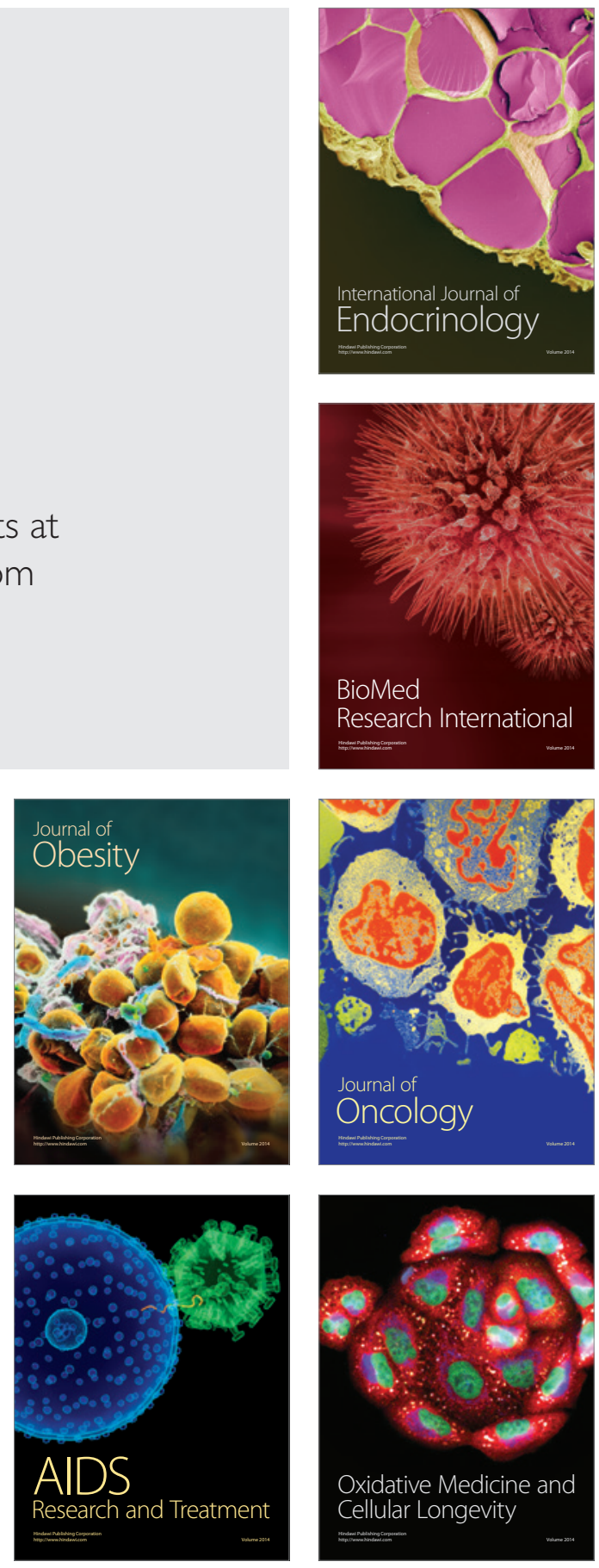